\title{
Effective temperatures of Ap stars
}

\author{
N.A. Sokolov ${ }^{1,2}$ \\ 1 Central Astronomical Observatory at Pulkovo, St. Petersburg 196140, Russia \\ e-mail: sokolov@gao.pnpi.spb.ru \\ 2 St. Petersburg State University, St. Petersburg, Russia
}

Received September 9; accepted December 3, 1997

\begin{abstract}
A new method of determination of the effective temperatures of Ap stars is proposed. The method is based on the fact that the slopes of the energy distribution in the Balmer continuum near the Balmer jump for "normal" main sequence stars and chemically peculiar stars with the same $T_{\text {eff }}$ are identical. The effective temperature calibration is based on a sample of main sequence stars with well known temperatures (Sokolov 1995). It is shown that the effective temperatures of Ap stars are derived by this method in good agreement with those derived by the infrared flux method and by the method of Stepien \& Dominiczak (1989). On the other hand, the comparison of obtained $T_{\text {eff }}$ with $T_{\text {eff }}$ derived from the color index (B2-G) of Geneva photometry shows a large scatter of the points, nevertheless there are no systematical differences between two sets of the data.
\end{abstract}

Key words: stars: chemically peculiar — stars: fundamental parameters

\section{Introduction}

In the last decade, the problem of the effective temperature determination of the chemically peculiar (CP) stars was considered by different authors. Two recent papers by Lanz et al. (1993) and by Hauck \& North (1993) contain the temperature calibrations of Geneva photometric indices for various kinds of $\mathrm{CP}$ stars. By $\mathrm{CP}$ stars, the authors mean the Am (CP1 stars) as well as the Ap stars, the latter including the He-wk (CP4 stars), MgMn (CP3 stars), Si, Cr and SrCrEu types (CP2 stars). These studies show once more the difficulty to derive photometrically the effective temperatures for most of the CP stars. Since the photometric methods are used, the color temperature is evidently derived and the color temperature is not always equal to the effective one. Therefore for any

Send offprint requests to: N.A. Sokolov (first address) peculiar stars the $T_{\text {eff }}$ determination from the photometry is much more complex than for normal stars owing to the fact that the colors of these stars can be affected by the blanketing effect.

In the literature there are a few determinations of $T_{\text {eff }}$ for CP stars independent of the photometry. The infrared flux method (IRFM) fist proposed by Blackwell \& Shallis (1977) was applied to five CP stars by Shallis \& Blackwell (1979) and to seven CP stars by Shallis et al. (1985). The method requires a measurement of the total integrated flux from the star at the Earth, an absolute flux at wavelength in the infrared region and the flux on the star's surface at the same wavelength. The latter flux is given by interpolation in Kurucz models. The ratio of the total integrated flux to the monochromatic one is used to determine the angular diameter and effective temperature simultaneously by means of an iterative procedure. This method is quite promising, because the difficulties resulting from blanketing could be discarded. Lanz (1985) and Glushneva (1987) determined the effective temperature for 28 Bp stars and for 13 peculiar B2 - F0 stars respectively by using IRFM. Glushneva found that effective temperatures obtained from $I$ and $J$ magnitudes were systematically lower up to $300 \mathrm{~K}$ than $T_{\text {eff }}$ obtained from $K$ magnitude. Meǵessier (1988) determined the effective temperatures for several standard stars in order to compare them with the fundamental values of Code et al. (1976) as well as for twelve CP2 stars by using the IRFM with integrated fluxes given by Lanz (1984). She got the closest agreement with Code et al. (1976) values, when restricting the method to $J$ and $K$ magnitudes. However, she found that the Hayes' (1979) calibration of the infrared photometry should be preferred.

Adelman (1985) estimated temperatures of 16 CP3 stars and 49 CP2 stars from fitting separately ultraviolet data, visual data and Balmer jump with the predictions of solar composition fully-line blanketed model atmospheres (Kurucz 1979). The comparison of the mean ultraviolet region temperatures shows substantial differences with 
visual color temperatures and Balmer jump temperatures. This may be due to the redistribution of the flux from the far-UV spectral region to the optical one.

Stepien \& Dominiczak (1989) suggested an independent method of $T_{\text {eff }}$ determination for chemically peculiar stars. This method assumes that the first order difference between normal and peculiar energy distributions is due to the extra blocking of the flux in the far-UV and the redistribution of it in the longer wavelengths. From the fit of observed visual energy distribution of CP stars with a solar-composition model, they derived the UV flux deficit relative to the model, and then the temperature correction to the model temperature.

The review of the various methods of $T_{\text {eff }}$ determination for the CP stars shows once more the difficulty of deriving the effective temperatures for these stars. Since one use the methods taking into consideration the blanketing effect, the obtained temperature is close to the effective one. In the infrared flux method a monochromatic flux is taken in the infrared region to minimize a blanketing effect. The method proposed by Stepien \& Dominiczak take into account the blanketing effect as well. The photometric methods may be useful, as it is possible to apply the correction of the color (or model) temperatures following the procedure proposed by Stepien \& Dominiczak and to give relatively good estimates of $T_{\text {eff }}$. Another way is to use the observational parameter which is not affected by peculiarities and can be applicable both to the "normal" main sequence stars and to CP stars. The Balmer continuum slope near the Balmer jump can be a useful tool for determination of $T_{\text {eff }}$ for CP stars (Sokolov 1995).

In this paper the determination of the effective temperatures of $\mathrm{CP}$ stars using the Balmer continuum slope near the Balmer jump is discussed. This study is based on the ground-based observations of the stars measured by Adelman, Pyper, Shore and White (Adelman et al. 1989) and by Pulkovo astronomers (Alekseeva et al. 1996), as described in Sect. 2. The determination of $T_{\text {eff }}$ from the Balmer continuum slope is described in Sect. 3. The principal results and discussion are presented in Sect. 4.

\section{Observational data and reductions}

\subsection{Original data}

The continuum energy distribution in the spectra of "normal" main sequence and chemically peculiar stars were taken from the catalog of stellar spectrophotometry (CSS). The observations reported in this catalog were made by Adelman, Pyper, Shore and White at Kitt Peak National Observatory, Mt. Wilson Observatory, and Palomar Observatory. The information on the telescopeinstrument combination and details of the reduction procedure are given by Adelman (1978). A machinereadable version of this catalog is available at the
Astronomical Data Center (ADC) and the Centre de Données Astronomiques de Strasbourg (CDS) (Adelman et al. 1989). This version includes the spectra of approximately 200 stars in the wavelength region from $3300 \AA$ to $7100 \AA$ A. For each star, both individual and average energy distributions are presented in this catalog. In the following analysis only average energy distributions are used, because most of $\mathrm{CP}$ stars are well known variable stars and, probably, the calculation of the Balmer continuum slope near Balmer jump from the average energy distribution would give the average value of $T_{\text {eff }}$ over the cycle of variability.

Recently, the final version of the Pulkovo spectrophotometric catalog of bright stars (PSC) in the range from $3200 \AA$ to $10800 \AA$ has been published (Alekseeva et al. 1996). This catalog is archived at the Pulkovo Observatory on a PC and consists of three parts: the first contains data for 602 stars in the wavelength region from $3200 \AA$ to $7350 \AA$, the second contains data for 285 stars in the wavelength region from $5000 \AA$ to $10800 \AA$ and the third contains the combine data from the preceding catalogues. All the catalogues are presented with a step of $25 \AA$ and with an accuracy of $1.5-2.0 \%$. The information on the instrumentation, observations and details of the reduction procedure is given by Alekseeva et al. (1996). In our study only the first part of the PSC is used.

\subsection{Correction for interstellar reddening}

Some of the investigated stars from the catalog of stellar spectrophotometry may be reddened. Adelman (1980) estimated the effect of interstellar reddening by using the $U B V$ photometry with the $Q$ method of Johnson \& Morgan (1953) and the synthesized $u-b$ and $b-y$ colors with the prescriptions of Crawford (1978). For the stars with $E(B-V)>0.03$, Adelman corrected the data for the effect of interstellar reddening by using of the Schild (1977) interstellar extinction law in combination with their adopted values of $E(B-V)$. In the cases when $E(B-V)>0.03$ for the investigated CP stars, the catalog of stellar spectrophotometry includes both the observed and the corrected for reddening average energy distributions. In our study for the stars with $E(B-V)>0.03$ the average energy distributions corrected for the interstellar reddening are used.

In order to estimate the effect of interstellar reddening of the investigated CP stars from the PSC the procedure described in the paper by Sokolov (1995) is used. It was controlled by the $U B V$ photometry according to the $Q$ method of Johnson \& Morgan (1953). The values of $E(B-V)<0.03$ were obtained for investigated $\mathrm{CP}$ stars of this catalog. Moreover, the stars under investigation being located at the distances of the order of $100 \mathrm{pc}$ or less from the Sun, hence the interstellar extinction was neglected. Therefore, the observed energy distributions of 


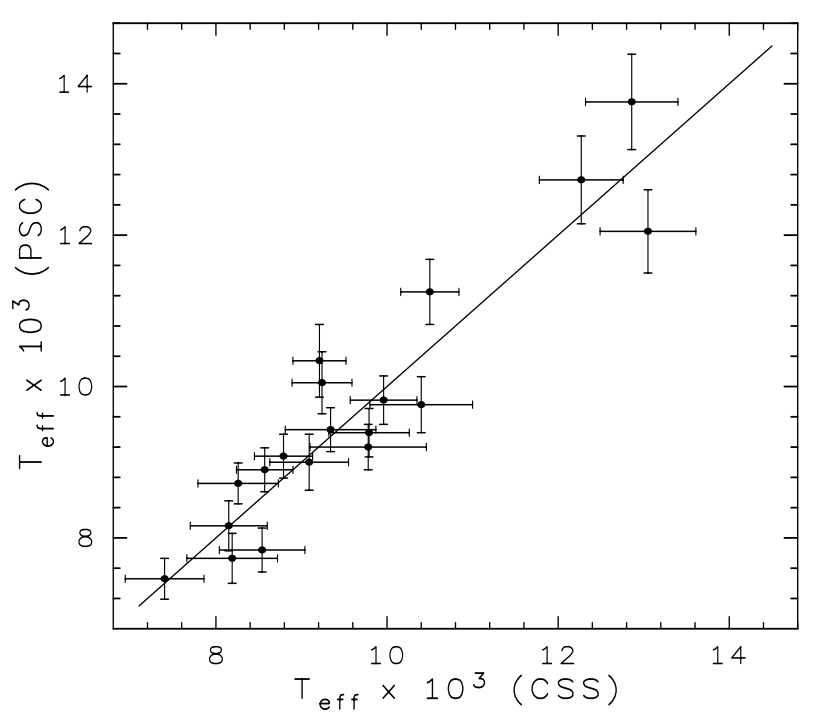

Fig. 1. Comparison of $T_{\text {eff }}$ derived from the Pulkovo spectrophotometric catalog of bright stars Alekseeva et al. (1996) with $T_{\text {eff }}$ derived from the catalog of stellar spectrophotometry (Adelman et al. 1989). The straight line denotes the relation $T_{\text {eff }}(\mathrm{CSS})=T_{\text {eff }}(\mathrm{PSC})$

the investigated CP stars from the PSC were not corrected for interstellar reddening. But, it should be noted that there is still the fundamental problem to separate the effects due to interstellar reddening from those due to the non-normal energy distributions of the CP stars.

\subsection{Flux calibration}

The catalog of stellar spectrophotometry (Adelman et al. 1989) was calibrated with the fluxes of the primary standard star Vega as presented by Hayes \& Latham (1975). On the other hand, the temperature calibration in the paper by Sokolov (1995) is established by using the stars calibrated with the absolute fluxes of Vega by Hayes (1985). To test the systematic differences between such various calibrations the method proposed by Sokolov (1995) has been applied to nineteen "normal" main sequence stars from the CSS and the computed temperatures were compared with $T_{\text {eff }}$ from the paper by Sokolov (1995). The comparison of the temperatures from two sources of the data shows no pronounced systematic discrepancies between them, as illustrated in Fig. 1. That is confirmed by the following results: the mean value of $\Delta T_{\text {eff }}=T_{\text {eff }}(\mathrm{PSC})-T_{\text {eff }}(\mathrm{CSS})$ is $67 \pm 138 \mathrm{~K}$ (the $\pm 138 \mathrm{~K}$ uncertainty refers to the rms standard deviation around the mean, devided by the square root of the number of stars), with a linear correlation coefficient $r=0.935$, and $\alpha=0.998$ for the regression line slope. The direct comparison of two calibrations of the absolute energy distribution for Vega given by Hayes (1985) and by Castelli \& Kurucz (1994) shows that the agreement between these calibrations is excellent (see Table 3 of Castelli
\& Kurucz 1994). Thus it is possible to use the catalog of stellar spectrophotometry by Adelman et al. (1989) for calculation of $T_{\text {eff }}$ from the slope of the Balmer continuum determined between $3300 \AA$ and $3600 \AA$ with temperature calibration of Sokolov (1995) without any correction for the differences between these flux calibrations.

The final list contains 68 chemically peculiar $\mathrm{Si}, \mathrm{Cr}$ and $\mathrm{SrCrEu}$ types (CP2 stars). One manganese star (36 Lyn) was also included, because this star was investigated both by Stepien \& Dominiczak (1989) and by Adelman (1985). Three stars in our list ( $\beta$ Tau, $\varepsilon$ UMa, HD 200311) have a double nature. The selected stars are listed in Table 1 together with their peculiarities and the number of individual scans.

\section{The method description}

In this section the method is proposed to derive the effective temperatures of CP stars by using the slope of the Balmer continuum near the Balmer jump. It is well known that the first order difference between energy distribution of "normal" main sequence stars and peculiar stars is caused by extra blocking of the flux in the far-UV and the redistribution of it in the longer wavelengths. The presented method implies the existence of a region of wavelengths between far-UV and visual regions with the same energy distribution for normal and peculiar stars. The comparison of the observed energy distributions with the best fitting models for twelve CP stars given by Stepien \& Dominiczak (1989) supports this assumption and shows that the slope of spectra in the Balmer continuum between $3200 \AA$ and $3600 \AA$ both for the observed energy distribution of CP stars and for the models is identical (see Fig. 2 of Stepien \& Dominiczak 1989). Only two stars HD 65339 and HD 125248 display the flux disagreement in this region of spectra, but the slopes of observed and theoretical energy distributions are similar for both stars.

From the theoretical point of view the flux in the Balmer continuum near the Balmer jump is formed in the outer layers of stellar atmosphere, because the absorption coefficient is high here and, probably, the effects caused by the peculiarity of CP stars are lesser. On the other hand, the Paschen continuum and the far-UV are formed in the inner layers where the effects of peculiarity of CP stars (the $\lambda 1400, \lambda 4200$ and $\lambda 5200$ broad continuum features, the photometric and magnetic field variability) are significant. The computations held by Leckrone et al. (1974) and by Muthsam $(1978,1979)$ show that there exists a wavelength $\lambda_{\operatorname{tr}}$ such that for $\lambda<\lambda_{\operatorname{tr}}$ the flux of radiation is suppressed relative to the flux of a normal star with the same effective temperature, whereas for $\lambda>\lambda_{\mathrm{tr}}$ it is enhanced. Note that the location of $\lambda_{\mathrm{tr}}$ is in the Balmer continuum near the Balmer jump, which is used for the determination of the effective temperatures (Sokolov 1995).

For determination of the shape of the continuum at both sides of the Balmer jump, an iterative procedure 
Table 1. Effective temperatures of CP2 (Ap magnetic) stars

\begin{tabular}{|c|c|c|c|c|c|c|c|c|c|}
\hline$\overline{\mathrm{HD}}$ & Name & Peculiarities & $\bar{~} n$ (vis.) & $\overline{\overline{T_{\text {eff }}\left(\Phi_{\mathrm{u}}\right)}}$ & $\overline{\sigma_{T_{\text {eff }}\left(\Phi_{\mathrm{u}}\right)}}$ & 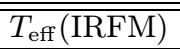 & Ref. & $\overline{\overline{T_{\text {eff }}(\mathrm{S} \& \mathrm{D})}}$ & $\overline{\overline{T_{\text {eff }}(\mathrm{B} 2-\mathrm{G})}}$ \\
\hline 1280 & $\theta$ And & $\mathrm{SiSr}$ & 7 & $8720^{*}$ & 270 & & & & 8930 \\
\hline 2453 & & $\mathrm{SrEuCr}$ & 5 & 8250 & 270 & & & 8880 & 8580 \\
\hline 4778 & GO And & $\mathrm{CrSrEu}$ & 55 & $9040^{*}$ & 280 & & & & 8930 \\
\hline 10783 & & $\mathrm{SiCrSr}$ & 8 & 10130 & 470 & & & 9980 & 9690 \\
\hline 14392 & 63 And & $\mathrm{Si}$ & 10 & 12210 & 500 & & & 11060 & 11350 \\
\hline 18296 & 21 Per & $\mathrm{SiSr}$ & 10 & 9370 & 370 & & & 9970 & 10580 \\
\hline 19832 & 56 Ari & $\mathrm{Si}$ & 18 & 12460 & 560 & & & 11600 & 11980 \\
\hline 25823 & 41 Tau & $\mathrm{SiSr}$ & 14 & 11750 & 500 & & & 12360 & 12720 \\
\hline 27309 & $56 \mathrm{Tau}$ & $\mathrm{Si}$ & 3 & 11000 & 480 & & & 11760 & 11840 \\
\hline 28319 & $78 \mathrm{Tau}$ & $\mathrm{Sr}$ & 4 & $7690 *$ & 320 & & & & \\
\hline 29305 & $\alpha$ Dor & $\mathrm{Si}$ & 3 & $12190^{*}$ & 550 & & & & \\
\hline 32633 & & $\mathrm{SiSr}$ & 14 & 10970 & 690 & & & 12100 & 10250 \\
\hline 32650 & BN Cam & $\mathrm{Si}$ & 3 & 12000 & 570 & & & 12020 & 11920 \\
\hline 34452 & IQ Aur & $\mathrm{Si}$ & 22 & 13870 & 760 & & & 14240 & 14990 \\
\hline 35497 & $\beta$ Tau & SiCrMn & 247 & $13410^{*}$ & 610 & 13824 & 5 & & 12120 \\
\hline 38104 & $o$ Aur & $\mathrm{SrEu}$ & 3 & 8910 & 440 & & & 8600 & 9060 \\
\hline 39317 & 137 Tau & $\mathrm{SiEuCr}$ & 3 & 9150 & 350 & & & 9600 & 9590 \\
\hline 40312 & $\theta$ Aur & $\mathrm{Si}$ & 19 & 10450 & 350 & 10060 & 4 & 10200 & 10250 \\
\hline 40394 & 36 Aur & $\mathrm{Si}$ & 3 & 9260 & 390 & & & 9280 & 9080 \\
\hline 43819 & V1155 Ori & $\mathrm{Si}$ & 4 & 10800 & 480 & 10775 & 3 & 10750 & 11100 \\
\hline 62140 & 49 Cam & $\mathrm{SrEu}$ & 3 & 8320 & 540 & & & 8000 & 7570 \\
\hline 64486 & & $\mathrm{Si}$ & 5 & 10510 & 520 & & & 10040 & 9940 \\
\hline 65339 & $53 \mathrm{Cam}$ & $\mathrm{SrCrEu}$ & 13 & 8430 & 740 & & & 9100 & 8190 \\
\hline 65575 & $\chi$ Car & $\mathrm{Si}$ & 4 & $20650^{*}$ & 1480 & & & & \\
\hline 68351 & $15 \mathrm{Cnc}$ & $\mathrm{SiCr}$ & 4 & 9650 & 430 & & & 9880 & 10040 \\
\hline 71866 & TZ Lyn & EuSrSi & 13 & 8270 & 590 & & & 8840 & 8660 \\
\hline 72968 & 3 Hya & $\mathrm{SrCr}$ & 3 & 9160 & 640 & & & 9310 & 9440 \\
\hline 74521 & $49 \mathrm{Cnc}$ & $\mathrm{SiEuSr}$ & 13 & 10230 & 490 & & & 10550 & 10100 \\
\hline 74535 & HX Vel & $\mathrm{Si}$ & 3 & $17380^{*}$ & 1320 & & & & \\
\hline 78045 & $\alpha \mathrm{Vol}$ & $\mathrm{Sr}$ & 4 & $8670^{*}$ & 260 & & & & 8290 \\
\hline 79158 & 36 Lyn & Mn & 3 & 12640 & 700 & & & 12480 & 12870 \\
\hline 90044 & 25 Sex & $\mathrm{SiCrSr}$ & 13 & 9770 & 600 & & & 10160 & 10220 \\
\hline 108945 & 21 Com & $\mathrm{Sr}$ & 3 & 8850 & 450 & & & 8800 & 8930 \\
\hline 112185 & $\varepsilon \mathrm{UMa}$ & CrEuMn & 25 & 9340 & 530 & 9470 & 4 & 9000 & 9530 \\
\hline 112413 & $\alpha^{2} \mathrm{CVn}$ & $\mathrm{SiCrEu}$ & 18 & 10410 & 510 & 10850 & 2 & & 11040 \\
\hline 116656 & $\zeta \mathrm{UMa}$ & $\mathrm{SrCr}$ & 10 & $8760^{*}$ & 260 & & & & \\
\hline 118022 & $78 \mathrm{Vir}$ & CrEuSr & 13 & 9250 & 580 & 9080 & 4 & 9400 & 9060 \\
\hline 119213 & CQ UMa & $\mathrm{SrCr}$ & 34 & $9000^{*}$ & 290 & & & & 8630 \\
\hline 120198 & $84 \mathrm{UMa}$ & $\mathrm{EuCr}$ & 4 & 9760 & 590 & & & 9890 & 9960 \\
\hline 124224 & CU Vir & $\mathrm{Si}$ & 49 & 12870 & 640 & 12775 & 3 & 12000 & 12220 \\
\hline 125248 & CS Vir & $\mathrm{EuCr}$ & 14 & 9290 & 520 & & & 9620 & 9250 \\
\hline 128898 & $\alpha \operatorname{Cir}$ & $\mathrm{SrEu}$ & 4 & $8440 *$ & 290 & & & & 7680 \\
\hline 133029 & BX Boo & $\mathrm{SiSrCr}$ & 12 & 11130 & 450 & & & 10700 & 10850 \\
\hline 135382 & $\gamma \operatorname{Tr} \mathrm{A}$ & $\mathrm{Eu}$ & 4 & $9030 *$ & 310 & & & & 9110 \\
\hline 137909 & $\beta \mathrm{CrB}$ & $\mathrm{SrEuCr}$ & 31 & 8150 & 450 & & & 8000 & 7430 \\
\hline 137949 & $33 \mathrm{Lib}$ & $\mathrm{SrEuCr}$ & 2 & 8420 & 490 & & & 7600 & \\
\hline 140160 & $\chi$ Ser & $\mathrm{Sr}$ & 18 & 8650 & 430 & 9150 & 2 & & 9120 \\
\hline 148330 & DQ Dra & $\mathrm{SiSr}$ & 2 & 9400 & 480 & & & 9200 & 9250 \\
\hline 155125 & $\eta \mathrm{Oph}$ & $\mathrm{Sr}$ & 13 & $8740^{*}$ & 310 & & & & 8900 \\
\hline 157792 & $44 \mathrm{Oph}$ & $\mathrm{Sr}$ & 4 & 9530 & 320 & & & & \\
\hline 165474 & & $\mathrm{SrCrEu}$ & 5 & 7800 & 320 & & & 7960 & \\
\hline 170973 & MV Ser & $\mathrm{SiCrSr}$ & 13 & 10050 & 570 & & & 9800 & \\
\hline 176232 & $10 \mathrm{Aql}$ & $\mathrm{Sr}$ & 10 & 8380 & 490 & 7700 & 2 & 7900 & 7620 \\
\hline 179527 & 19 Lyr & $\mathrm{Si}$ & 3 & 10320 & 370 & & & 10150 & 9660 \\
\hline 182640 & $\delta \mathrm{Aql}$ & $\mathrm{Ca}-\mathrm{w}$ & 3 & $6880^{*}$ & 230 & & & & 7100 \\
\hline 183056 & 4 Cyg & $\mathrm{Si}$ & 3 & 12030 & 470 & & & 11800 & 10960 \\
\hline 184905 & & $\mathrm{SiCr}$ & 2 & 10030 & 610 & & & 10270 & 10100 \\
\hline
\end{tabular}


Table 1. continued

\begin{tabular}{|c|c|c|c|c|c|c|c|c|c|}
\hline HD & Name & Peculiarities & $n$ (vis.) & $\overline{T_{\text {eff }}\left(\Phi_{\mathrm{u}}\right)}$ & $\overline{\sigma_{T_{\text {eff }}\left(\Phi_{\mathrm{u}}\right)}}$ & $T_{\text {eff }}($ IRFM $)$ & Ref. & $\bar{T}_{\text {eff }}(\mathrm{S} \& \mathrm{D})$ & $\overline{T_{\text {eff }}(\mathrm{B} 2-\mathrm{G})}$ \\
\hline 192678 & & $\mathrm{Sr}$ & 3 & 9150 & 350 & & & 9040 & 9270 \\
\hline 196178 & & $\mathrm{Si}$ & 3 & 12830 & 530 & & & 12700 & 11710 \\
\hline 196524 & $\beta$ Del & $\mathrm{Sr}$ & 11 & $6240^{*}$ & 270 & & & & 6510 \\
\hline 200311 & & $\mathrm{SiCrHg}$ & 7 & 11740 & 610 & & & 12300 & 11240 \\
\hline 201601 & $\gamma \mathrm{Equ}$ & $\mathrm{SrCrEu}$ & 8 & 8430 & 440 & 7610 & 1 & & 7630 \\
\hline 204411 & & $\mathrm{Cr}$ & 14 & 8250 & 380 & 8750 & 2 & 8500 & 8770 \\
\hline 205087 & & $\mathrm{SrSiCr}$ & 16 & 10270 & 540 & & & 10460 & 10080 \\
\hline 206088 & $\gamma$ Cap & $\mathrm{Sr}$ & 6 & $6590^{*}$ & 220 & & & & 7250 \\
\hline 206742 & $\iota \mathrm{PsA}$ & $\mathrm{Si}$ & 9 & 10990* & 400 & & & & 9860 \\
\hline 220825 & $\kappa \operatorname{Psc}$ & $\mathrm{CrSrEu}$ & 10 & 8730 & 450 & 9250 & 2 & 9500 & 9070 \\
\hline 223640 & $108 \mathrm{Aqr}$ & $\mathrm{SiSrCr}$ & 4 & 12180 & 470 & 12390 & 3 & 12200 & 11930 \\
\hline
\end{tabular}

References: 1. Shallis \& Blackwell (1979), 2. Shallis et al. (1985), 3. Mégessier (1988), 4. Glushneva (1987), 5. Underhill et al. (1979).

was used that permits to exclude absorption lines, as described by Sokolov (1995). The values of the Balmer continuum parameters were computed by using the linear approximation in the form $\Delta m_{\lambda}=\Psi_{\mathrm{u}}+\Phi_{\mathrm{u}} \cdot(1 / \lambda)$. Here $\Delta m_{\lambda}=m_{\lambda}-m_{5550 \AA} \AA$, the parameter $\Phi_{\mathrm{u}}$ is the slope of the Balmer continuum and $\Psi_{\mathrm{u}}$ is the constant term of the linear approximation. In the paper by Sokolov (1995) it is shown that the slope of the Balmer continuum between $3200 \AA$ and $3600 \AA$ is a very good indicator of the effective temperature for $\mathrm{B}, \mathrm{A}$ and $\mathrm{F}$ main sequence stars. The linear calibration curve between $\Theta_{\text {eff }}=5040 / T_{\text {eff }}$ and $\Phi_{\mathrm{u}}$ is established by using the standard stars with well known temperatures taken from Code et al. (1976) and Malagnini et al. (1986). This temperature calibration can be applied in the wide temperature interval from $6000 \mathrm{~K}$ to $27000 \mathrm{~K}$.

Based on the theoretical prediction, as well as on the fact that the slope of the Balmer continuum near the Balmer jump of models and CP stars is identical, this observational parameter is used for determination of the effective temperatures of CP stars. The temperature calibration of B, A and F main sequence stars (Sokolov 1995) is applied to the $\mathrm{CP}$ stars, which was defined as:

$$
\Theta_{\text {eff }}=0.605+0.334 \cdot \Phi_{\mathrm{u}} \text {. }
$$$$
\pm 0.015 \pm 0.007 \text {. }
$$

The errors on effective temperatures are computed by taking into account the contributions from different terms, according to the errors' formula for the standard propagation theory:

$\sigma_{T_{\mathrm{eff}}}=A \cdot\left[\sigma_{\mathrm{a}}^{2}+\Phi_{\mathrm{u}}^{2} \sigma_{\mathrm{b}}^{2}+b^{2}\left(\sigma_{\Phi}^{2}+E^{2} \sigma_{\mathrm{S}}^{2}+S^{2} \sigma_{\mathrm{E}}^{2}\right)\right]^{\frac{1}{2}}$

where $A=T_{\text {eff }}{ }^{2} / 5040, \sigma_{\mathrm{a}}$ and $\sigma_{\mathrm{b}}$ refer to the errors of the calibration coefficients and $\sigma_{\Phi}$ is the error in the slope of the energy distribution. The values of errors for the mean interstellar extinction law $-\sigma_{\mathrm{S}}$ and for the colour excess $E(B-V)-\sigma_{\mathrm{E}}$ are estimated in the paper (Sokolov 1995) and are equal to 0.048 and 0.03 , respectively.

Generally, the least-squares procedure is used in order to compute the values of the Balmer continuum parameters near the Balmer jump. The effective temperatures of the CP stars are derived from the slope of spectra in the Balmer continuum by using Eq. (1). The errors on $T_{\text {eff }}$ were computed by using Eq. (2). It should be noted, that for the stars with $E(B-V)<0.03$, only the errors in the calibration coefficients and the error in the slope of the energy distribution are taken into account. For stars with $E(B-V)>0.03$ the errors on $T_{\text {eff }}$ were computed taking into account additionally the values $\sigma_{\mathrm{S}}$ and $\sigma_{\mathrm{E}}$. For these stars the average energy distribution corrected for interstellar reddening was used from the catalog of stellar spectrophotometry.

\section{Results and discussion}

The previously described calibration curve was applied to 50 CP2 stars from the catalog of stellar spectrophotometry (Adelman et al. 1989) and to 18 CP2 stars from the Pulkovo spectrophotometric catalog of bright stars (Alekseeva et al. 1996). For these stars the HD number, name, peculiarity according to General Catalogue of Ap and Am stars by Renson et al. (1991), number of the visual scans, temperature and error obtained from the relationships (1) and (2), respectively are given in Table 1. An asterisk following $T_{\text {eff }}\left(\Phi_{\mathrm{u}}\right)$ means that this datum was computed from energy distribution of the PSC.

To test the validity of the proposed method of determination of $T_{\text {eff }}$ for CP2 stars, the temperatures derived from $\Phi_{\mathrm{u}}$ were compared with those derived by the infrared flux method, by the Stepien \& Dominiczak (1989) method and with temperatures derived from (B2-G) color index of Geneva photometry.

\subsection{A comparison with infrared flux method determinations}

In the literature there are five papers concerning the CP2 stars where the effective temperatures are derived by the infrared flux method. These papers are those by Shallis 


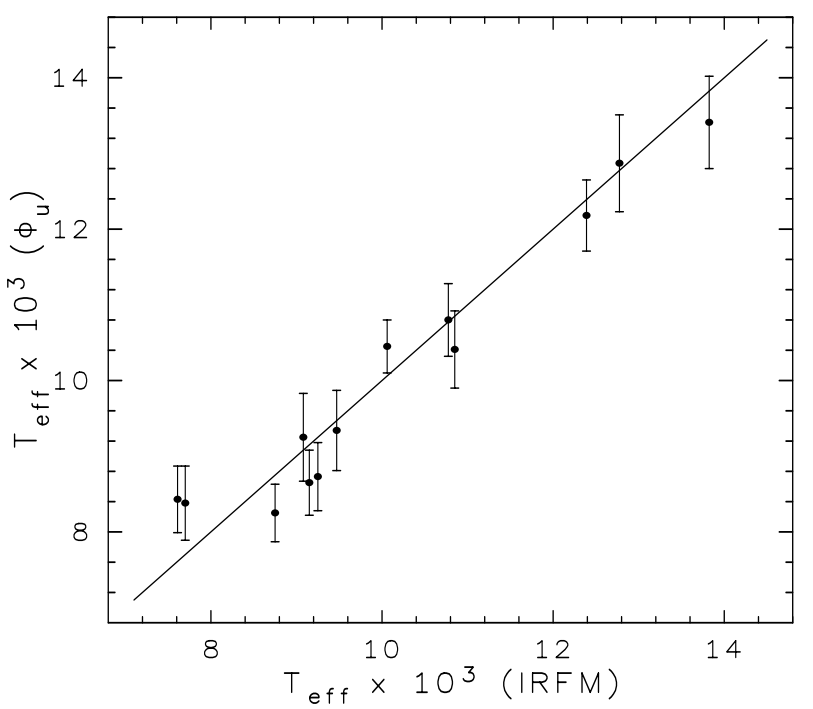

Fig. 2. Comparison of $T_{\text {eff }}$ derived from $\Phi_{\mathrm{u}}$ with those derived by infrared flux method

\& Blackwell (1979), Shallis et al. (1985), Lanz (1985), Glushneva (1987), Meǵessier (1988). The star HD 35497 ( $\beta \mathrm{Tau})$ is mentioned in the paper by Underhill et al. (1979), where the effective temperatures are derived by the same way. The effective temperature for this star derived by Underhill et al. was taken into consideration as well. If there are several determinations of $T_{\text {eff }}$ by IRFM for individual star then the latest determination is considered. For example, all the CP2 stars investigated by Lanz are mentioned in Mégessier's paper. For the common stars with those of Table 1 , their $T_{\text {eff }}$ values and the references are given in Cols. 7 and 8 , respectively. The values of $T_{\text {eff }}$ derived from $\Phi_{\mathrm{u}}$ are compared with those obtained by IRFM for 13 common stars (see Fig. 2, where the straight line denotes the relation $\left.T_{\text {eff }}(\operatorname{IRFM})=T_{\text {eff }}\left(\Phi_{\mathrm{u}}\right)\right)$. One can see, that the agreement appears to be very good. It is confirmed by the following results: $\Delta T_{\text {eff }}=41 \pm 127 \mathrm{~K}, r=0.972, \alpha=0.904$. Only for two cool stars: HD 176232 (10 Aql) and HD 201601 ( $\gamma$ Equ) the temperatures derived by Shallis et al. (1985) and by Shallis \& Blackwell (1979), respectively, are relatively low. Glushneva (1987) has noted the same disagreement with Shallis \& Blackwell (1979) determinations. So she has redetermined the effective temperature of the star HD 112185 ( $\varepsilon \mathrm{UMa}$ ), obtaining a value of $9470 \mathrm{~K}$, which is higher than that of $8920 \mathrm{~K}$ by Shallis \& Blackwell. For this star the effective temperature derived from $\Phi_{\mathrm{u}}$ is equal $9340 \mathrm{~K}$, this value is in agreement with the temperature obtained by Glushneva. Hauck \& North (1993) also noted that the temperature derived by Shallis \& Blackwell (1979) for the star HD 358 ( $\alpha$ And) is relatively low. Finally, the effective temperatures derived from $\Phi_{\mathrm{u}}$ (with the exception of two cool stars) are in the excellent agreement with $T_{\text {eff }}$ obtained by IRFM.

\subsection{A comparison with the determinations obtained by the method of Stepien \& Dominiczak}

Stepien \& Dominiczak (1989) proposed the new method to determine the effective temperatures of CP2 stars. From a fit of the observed visual energy distribution of $\mathrm{CP}$ stars with a solar-composition model, they derived the flux deficit relative to the model, and then a temperature correction to the model temperature. Practically, the method includes two steps: first, the model temperature $T_{\mathrm{M}}$ is found from the detailed fit of the Kurucz's model to the observed visual energy distribution and second, the obtained $T_{\mathrm{M}}$ should be transformed to $T_{\text {eff }}$ by using the relation, defined by Stepien \& Dominiczak as:

$T_{\text {eff }}=1600+0.80 \cdot T_{\mathrm{M}}$.

They derived this relation by using twelve CP stars with available visual and UV energy distributions. In order to have wider sample of stars for the comparison, this method was applied to the model temperatures obtained by Adelman (1985). He derived temperatures for 49 CP2 stars from a fit of the Kurucz's model to the observed ultraviolet and visual energy distributions as well as to the Balmer jump and received three temperatures: $T(\mathrm{UV})$, $T(\mathrm{PC})$ and $T(\mathrm{BJ})$ for each star. All the CP2 stars investigated by Stepien \& Dominiczak are presented in Adelman's paper. This allows to compare the Paschen continuum temperature - $T(\mathrm{PC})$ and the Balmer jump temperature - $T(\mathrm{BJ})$ derived by Adelman with the $T_{\mathrm{M}}$ derived by Stepien \& Dominiczak. For five stars the values of $T(\mathrm{PC})$ and $T(\mathrm{BJ})$ are equal and in the good agreement with $T_{\mathrm{M}}$. But, the comparison of all 12 stars shows that the $T_{\mathrm{M}}$ is $319 \mathrm{~K}$ cooler than the $T(\mathrm{BJ})$ and $208 \mathrm{~K}$ hotter than $T(\mathrm{PC})$. On the other hand, the computed as a mean value from the $T(\mathrm{PC})$ and the $T(\mathrm{BJ})$ model temperatures are in good agreement with $T_{\mathrm{M}}$ derived by Stepien \& Dominiczak. The mean difference between two sets of data is equal to $111 \mathrm{~K}$.

Based on this temperature comparison as well as on the conclusions given by Mégessier (1988) and by Stepien \& Dominiczak (1989) on importance of the Balmer jump in the determination of the temperature, the values of $T_{\mathrm{M}}$ as mean from the $T(\mathrm{PC})$ and the $T(\mathrm{BJ})$ were calculated for all stars of Table 2 from paper of Adelman (1985). After that, this mean value of $T_{\mathrm{M}}$ was corrected for the blanketing effect by using Eq. (3). The resulting temperatures $\left(T_{\text {eff }}(\mathrm{S} \& \mathrm{D})\right)$ are reported in Col. 9 of Table 1. Figure 3 gives a plot of $T_{\text {eff }}$ derived from $\Phi_{\mathrm{u}}$ versus $T_{\text {eff }}(\mathrm{S} \& \mathrm{D})$ for 47 common stars. The straight line corresponds to the relation $T_{\text {eff }}\left(\Phi_{\mathrm{u}}\right)=T_{\text {eff }}(\mathrm{S} \& \mathrm{D})$. As one can see from Fig. 3, for most of the stars the agreement between $T_{\text {eff }}\left(\Phi_{\mathrm{u}}\right)$ and $T_{\text {eff }}(\mathrm{S} \& \mathrm{D})$ appears to be very good, but for some stars the difference between two determinations is more than one sigma error. This discrepancy can be the result of the errors in the computation of the mean value of $T_{\mathrm{M}}$ from the $T(\mathrm{PC})$ and the $T(\mathrm{BJ})$ or the value of $\Phi_{\mathrm{u}}$ in our method for individual stars. The effect of variability 


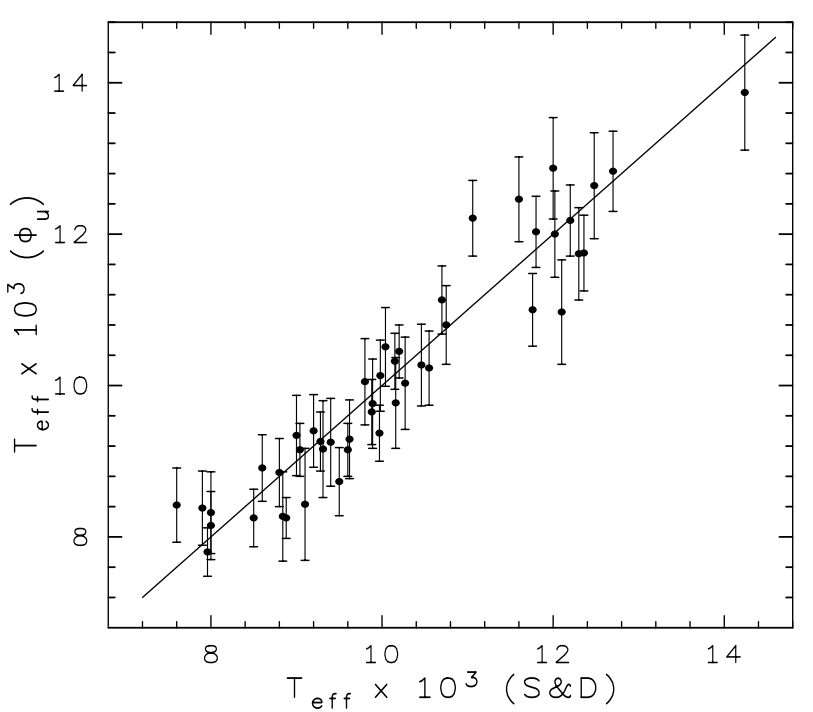

Fig. 3. Comparison of $T_{\text {eff }}$ derived from $\Phi_{\mathrm{u}}$ with those derived by the method proposed by Stepien \& Dominiczak (1989)

of these CP2 stars should be excluded, because for both methods the same observed energy distribution was used. Basically, there are no systematical differences between two sets of the data that is confirmed by the following results: $\Delta T_{\text {eff }}=38 \pm 69 \mathrm{~K}, r=0.952, \alpha=0.965$.

\subsection{A comparison with photometric determinations}

The photometric methods to determine $T_{\text {eff }}$ of CP stars were proposed in many photometric systems. As a rule, these methods are based on colors in the visible region of spectrum, thus it is necessary to correct the color temperature for blanketing effect to obtain an effective one. Comparisons of $T_{\text {eff }}$ derived by IRFM or by the method of Stepien \& Dominiczak with $T_{\text {eff }}(B 2-G)$ and $T_{\text {eff }}(X, Y)$ given by Hauck \& North (1993) show that $(B 2-G)$ color index is a very good estimator of $T_{\text {eff }}$ for CP2 stars. Moreover the color index $(B 2-G)$ can be used without a correction for the blanketing effect, because this effect is included in the temperature calibration, while the reddening - free parameters $X$ and $Y$ should be corrected for that. It should be noted, that the $(B 2-G)$ color index is to be corrected for the interstellar reddening, but the parameters $X$ and $Y$ are reddening-free. In order to estimate photometrically the effective temperatures of CP2 stars the $(B 2-G)$ color index was used. The values of $T_{\text {eff }}(B 2-G)$ were taken from Table 1 of Hauck \& North paper for the sample of 21 common stars. In addition, the values of $T_{\text {eff }}(B 2-G)$ for the 38 stars are computed according to the procedure described by Hauck \& North (1993) and by using the photometric data from the paper by Hauck \& North (1982). The values of $T_{\text {eff }}(B 2-G)$ for the 59 common stars are presented in Col. 10 of Table 1. Figure 4 gives a plot of the $T_{\text {eff }}$ derived

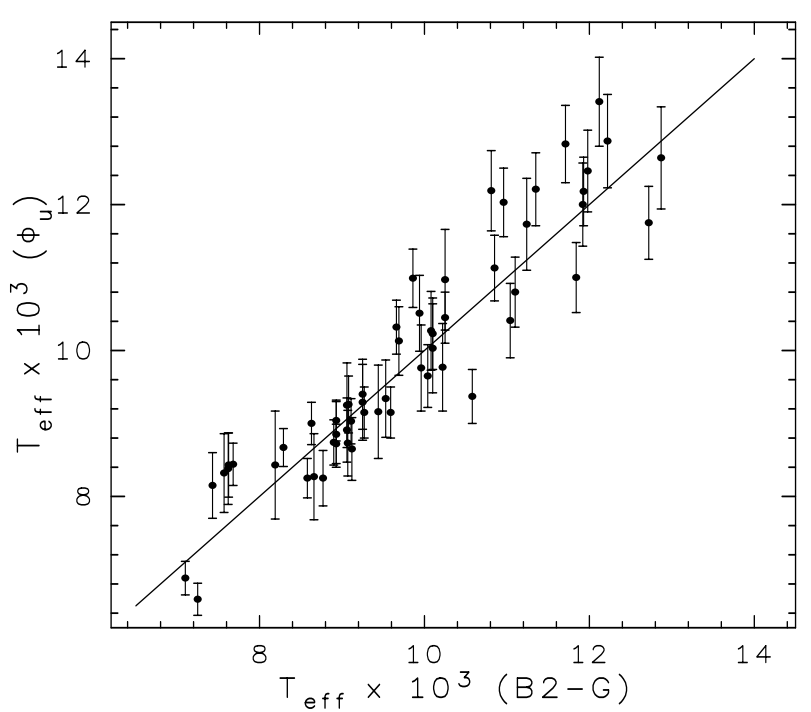

Fig. 4. Comparison of $T_{\text {eff }}$ derived from $\Phi_{\mathrm{u}}$ with those derived from $(B 2-G)$ color index of Geneva photometry

from $\Phi_{\mathrm{u}}$ versus $T_{\mathrm{eff}}(B 2-G)$. One can see, there are no systematic differences between two sets of the data, though the scattering of the points in Fig. 4 is high enough (up to $1000 \mathrm{~K}$ ), especially for the stars with $T_{\text {eff }}>9500 \mathrm{~K}$. So, the comparison of $T_{\text {eff }}$ derived from $\Phi_{\mathrm{u}}$ with $T_{\text {eff }}(B 2-G)$ shows that for 28 stars with $T_{\text {eff }} \leq 9500 \mathrm{~K}$ the agreement between two sets of data is very good. The mean difference between $T_{\text {eff }}\left(\Phi_{\mathrm{u}}\right)$ and $T_{\text {eff }}(B 2-G)$ is $42 \pm 80 \mathrm{~K}$. For all the stars in our sample the mean effective temperature difference is $\Delta T_{\text {eff }}=T_{\text {eff }}\left(\Phi_{\mathrm{u}}\right)-T_{\text {eff }}(B 2-G)=102 \pm 76 \mathrm{~K}$, with a linear correlation coefficient $r=0.938$, and $\alpha=0.975$ for the slope of the regression line of $T_{\text {eff }}\left(\Phi_{\mathrm{u}}\right)$ versus $T_{\text {eff }}(B 2-G)$. The comparison of the stars with $T_{\text {eff }}>$ $9500 \mathrm{~K}$ shows that $T_{\text {eff }}(B 2-G)$ are slightly less than $T_{\text {eff }}\left(\Phi_{\mathrm{u}}\right)$. For these stars the mean difference $\Delta T_{\text {eff }}$ is equal $154 \pm 124 \mathrm{~K}$. Probably this difference can be explained by the correction of $(B 2-G)$ color index for interstellar reddening because it is very hard to separate the effects due to interstellar reddening from those due to the non-normal energy distribution of CP2 stars. It is also confirmed by the conclusion of Hauck \& North (1993) that for CP2 stars with $T_{\text {eff }}>9500 \mathrm{~K}$ the values of $T_{\text {eff }}(B 2-G)$ are less reliable. For these stars they propose to use the reddening-free parameters $X$ and $Y$.

The $X$ and $Y$ parameters of Geneva photometry are primarily related to $T_{\text {eff }}$ and $\log g$ respectively, but they can be affected by the stars's peculiarity, especially for CP2 stars cooler than $11000 \mathrm{~K}$ (North \& Cramer 1984). However, for CP2 stars Hauck \& North (1993) propose to use the $X$ and $Y$ parameters and transform $T_{\text {eff }}(X, Y)$ to $T_{\text {eff }}$ through relation between $T_{\text {eff }}(X, Y)$ and $T_{\text {eff }}$ obtained by these authors. On the other hand, according to results presented by Lanz et al. (1993) the significant scattering (up to $2000 \mathrm{~K}$ ) can be observed for some values of $X$ and the uncertainties in temperatures are rather large (about 
$1000 \mathrm{~K})$. In other words, the $X$ parameter may give the same scattering of points when comparing $T_{\text {eff }}\left(\Phi_{\mathrm{u}}\right)$ with $T_{\text {eff }}(X)$.

Generally, there are no significant systematical differences between the temperatures derived from $\Phi_{\mathrm{u}}$ and those derived from fluxes by other methods. The temperature calibration derived for B, A and F main sequence stars is applicable to CP2 stars as well. The temperature derived from $\Phi_{\mathrm{u}}$ for CP2 stars may be identified with their effective one, because the influence of the stars's peculiarity on the Balmer continuum slope near the Balmer jump is negligible.

Taking into account the observational uncertainties of the slope, the calibration errors, as well as the errors for the mean interstellar extinction law and for the value $E(B-V)$ for the reddened stars the error of $T_{\text {eff }}$ are calculated for each star of our sample. The statistical errors of the temperature determination vary from star to star with their values ranging from $5 \%$ to $10 \%$. As a rule, the large errors in the temperature determination related to the stars with large photometric variations.

In our study only $\mathrm{Si}, \mathrm{Cr}$ and $\mathrm{SiCrEu}$ types of $\mathrm{CP}$ stars were used, but this method can be extended to other types of CP stars when a blanketing effect is less and the temperature derived from $\Phi_{\mathrm{u}}$ should be close to the effective one. For example, Hauck \& North (1993) have shown that the colour temperature of $\mathrm{CP} 1(\mathrm{Am})$ and of $\mathrm{CP} 4(\mathrm{He}-\mathrm{wk})$ stars may be identified with their effective temperature. In the case of CP3 (MgMn) stars it is less clear, because for these stars there are a few of direct $T_{\text {eff }}$ determinations. The fact that their UV flux is normal (Jamar et al. 1978) suggests that the colour temperature of CP3 stars may be also identified with their effective one.

\section{Conclusions}

The present study shows that the slope of the Balmer continuum near the Balmer jump can be a very good estimator of $T_{\text {eff }}$ for CP2 stars. The $T_{\text {eff }}$ for $68 \mathrm{CP}$ stars was determined from the continuum near the Balmer jump by using the average energy distribution of the stars measured by Adelman, Pyper, Shore and White and by Pulkovo astronomers as well. From comparison of $T_{\text {eff }}\left(\Phi_{\mathrm{u}}\right)$ with $T_{\text {eff }}$ derived by various methods and by different sets of observations it is concluded that there is a good agreement between the effective temperatures for the subsets of common stars.

There is a clear need for the further applications of this method to CP1, CP3 and CP4 stars, to have a large amount of data from which it would be possible to analyse the problems of effective temperature, classification and atmosphere modeling of CP stars. There is also a need to study the effective temperature and the Balmer jump variations with a phase for stars with enough number of the individual scans.
Acknowledgements. The author would like to thank Drs. V.A. Hagen-Thorn, I.M. Kopylov and I.S. Guseva for their helpful comments. This work has been supported by the Russian National Foundation for Astronomy (project No. 1.4.1.2).

\section{References}

Adelman S.J., 1978, ApJ 222, 547

Adelman S.J., 1980, A\&AS 42, 375

Adelman S.J., 1985, PASP 97, 970

Adelman S.J., Pyper D.M., Shore S.N., White R.E., Warren W.H., 1989, A\&AS 81, 221

Alekseeva G.A., Arkharov A.A., Galkin V.D., et al., 1996, Baltic Astron. 5, 603

Blackwell D.E., Shallis M.J., 1977, MNRAS 180, 177

Castelli F., Kurucz R.L., 1994, A\&A 281, 817

Code A.D., Davis J., Bless R.C., Hanbury Brown R., 1976, ApJ 203, 417

Crawford D.L., 1978, AJ 83, 48

Glushneva I.N., 1987, AZh 64, 601

Hauck B., North P., 1982, A\&A 114, 23

Hauck B., North P., 1993, A\&A 269, 403

Hayes D.S., Latham D.W., 1975, ApJ 197, 593

Hayes D.S., 1979, Dudley Obs. Rep. 14, 297

Hayes D.S.: 1985, Stellar Absolute Fluxes and Energy Distribution from 0.32 to $4.0 \mu \mathrm{m}$. In: IAU Symp. 111, Calibration of Fundamental Stellar Quantities, Hayes D.S., Pasinetti L.E., Davis Philip A.G. (eds.). Reidel Dordrecht, p. 225

Jamar C., Macau-Hercot D., Praderie F., 1978, A\&A 63, 155

Johnson H.L., Morgan W.W., 1953, ApJ 117, 313

Kurucz R.L., 1979, ApJS 40, 1

Lanz T., 1984, A\&A 139, 161

Lanz T., 1985, A\&A 144, 191

Lanz T., Artru M.C., Didelon P., Mathys G., 1993, A\&A 272, 465

Leckrone D.S., Fowler J.W., Adelman S.J., 1974, A\&A 32, 237

Malagnini M.L., Morossi C., Rossi L., Kurucz R.L., 1986, A\&A 162,140

Mégessier C., 1988, A\&AS 72, 551

Muthsam H., 1978, A\&AS 35, 107

Muthsam H., 1979, A\&A 73, 159

North P., Cramer N., 1984, A\&AS 58, 387

Renson P., Gerbaldi M., Catalano F.A., 1991, A\&AS 89, 429

Schild R.E., 1977, AJ 82, 337

Shallis M.J., Blackwell D.E., 1979, A\&A 79, 48

Shallis M.J., Baruch J.E.F., Booth A.J., Selby M.J., 1985, MNRAS 213, 307

Sokolov N.A., 1995, A\&AS 110, 553

Stepien K., Dominiczak R., 1989, A\&A 219, 197

Underhill A.B., Divan L., Prévot-Burnichon M.L., Doazan V., 1979, MNRAS 189, 601 